\title{
FIRST-ORDER OPTICAL PHONON PROCESSES IN AMORPHOUS CLUSTERS
}

\author{
M.A. GRADO CAFFARO, consultant (Madrid, Spain) and \\ M. GRADO CAFFARO, consultant (Madrid, Spain) \\ C./Julio Palacios, 11, 9B, 28029-Madrid (Spain) \\ (Received August 10, 1993; in final form September 10, 1993)
}

Optical absorption in the far infrared range for amorphous $\mathrm{GaAs}, \mathrm{SiC}$ and $\mathrm{Ge}$ is investigated. Small clusters in the context of dynamical disorder are considered. Average values of the absorption contributions due to both dynamical and structural disorders are introduced. In particular, an equation for the spectrum due to dynamical disorder in amorphous $\mathrm{SiC}$ is presented.

\section{INTRODUCTION}

In the following, we shall refer to silicon carbide, germanium and III-V semiconductors in their amorphous state. Certain considerations on the above materials are also valid for other amorphous semiconductors. We shall study both dynamical and structural disorders by considering average values of optical absorption in the far infrared range.

Recently, both theoretical and experimental work on amorphous $\mathrm{SiC}$ has been developed by using a conventional methodology. We shall analyse $\mathrm{SiC}$ behavior in its amorphous state in a special way so that comparison of this behavior with the optical properties of $\mathrm{Ge}, \mathrm{Si}$ and $\mathrm{GaAs}$ is feasible.

\section{THEORETICAL FORMULATION}

First, let us consider small clusters in amorphous GaAs. M.A. Grado and M. Grado have obtained recently the following expression for dynamical disorder: ${ }^{1}$

$\xi_{2}^{(\mathrm{d})}(\omega) \approx \operatorname{ar}_{\mathrm{o}}\left(1-\frac{1}{3} \mathrm{br}_{\mathrm{o}}^{2}\right)\left(\xi_{\mathrm{o}}-\xi_{\infty}\right) \omega_{\mathrm{TO}(\mathrm{c})} \gamma_{\mathrm{c}}^{-1} \omega$

To derive eq. (1) a small cluster is assumed, so that the "length" of the cluster, $r_{\mathrm{o}}$, is small. Moreover, we have considered $\omega_{\text {TO }}=\omega ; \omega_{\text {TO }}$ stands for the angular frequency of the transversal optical mode and we have supposed that $\omega_{\text {TO }}$ does not depend approximately on $r$ (distance) in the cluster considered. In eq. (1) a and $\mathrm{b}$ are constants $(\mathrm{a}>0 ; \mathrm{b}>0), \xi_{\mathrm{o}}$ is the value of the imaginary part of the static 
dielectric constant, $\xi_{\infty}$ is the value of the imaginary part of the high-frequency dielectric constant, $\omega_{\mathrm{TO}(\mathrm{c})}$ is the angular frequency of the transversal optical mode in the crystalline state, and $\gamma_{\mathrm{c}}$ is a damping factor.

On the other hand, the spectrum due to structural disorder is given by ${ }^{1-3}$.

$\xi_{2}^{(\mathrm{s})}(\omega) \approx \mathrm{Kg}(\omega)$

where $\mathrm{K}$ is a constant and $\mathrm{g}(\omega)$ is the phonon density of states. Eq. (2) is valid for the far infrared range. Hereafter, we shall consider this range only.

Then, total spectrum is given by

$\xi_{2}(\omega) \approx \mathrm{Kg}(\omega)+\mathrm{K}^{\prime} \omega$

where $K^{\prime}$ is a constant namely:

$\mathrm{K}^{\prime}=\operatorname{ar}_{\mathrm{o}}\left(1-\frac{1}{3} \mathrm{br}_{\mathrm{o}}^{2}\right)\left(\xi_{\mathrm{o}}-\xi_{\infty}\right) \gamma_{\mathrm{c}}^{-1} \omega_{\mathrm{TO}(\mathrm{c})}$

and $\xi_{2}(\omega)$ is given by ${ }^{1,4,5}$

$\xi_{2}(\omega) \approx \mathrm{K} \int_{0}^{\hbar \omega} \mathrm{g}(\mathrm{E}) \mathrm{g}(\hbar \omega-\mathrm{E}) \mathrm{dE}$

for $\hbar \omega>2 \mathrm{E}_{c} ; \hbar$ is the modified Planck constant $(\hbar \equiv \mathrm{h} / 2 \pi)$, E stands for electronic energy, and $E_{c}$ is the energy at the conduction band edge. Eq. (4) can be expressed as a convolution, namely:

$$
\xi_{2}(\omega) \approx \mathrm{K}[\mathrm{g}(\omega)] *[\mathrm{~g}(\omega)]
$$

Hereafter, we will consider the case in which $\hbar \omega>2 \mathrm{E}_{\mathrm{c}}$ exclusively. By applying Laplace transform in eq: (5) we get:

$\mathrm{g}(\omega)=\mathrm{K}^{-1 / 2} £^{-1}\left\{\left\{£\left[\xi_{2}(\omega)\right]\right\}^{1 / 2}\right\}$

where $£$ stands for the Laplace transform operator.

Next we will take average values in both sides of eq. (3) by taking into account eq. (4). We get

$\int_{0}^{\Omega} \mathrm{d} \omega \int_{0}^{\omega} \mathrm{g}(\mathrm{E}) \mathrm{g}(\omega-\mathrm{E}) \mathrm{dE} \approx \int_{0}^{\Omega} \mathrm{g}(\omega) \mathrm{d} \omega+\frac{1}{2} \frac{\mathrm{K}^{\prime}}{\mathrm{K}} \Omega^{2}$

where $\Omega$ is the maximum angular frequency in the far infrared range; for convenience, we have taken $\hbar=1$ (atomic units). Eq. (7) is an interesting relationship in terms of the phonon density of states. From this equation we consider the following average values:

$\left\langle\xi_{2}^{(\mathrm{d})}(\omega)\right\rangle=\frac{1}{2} \mathrm{~K}^{\prime} \Omega$ 
$\left\langle\xi_{2}^{(s)}(\omega)\right\rangle=\mathrm{K} \Omega^{-1} \int_{0}^{\Omega} \mathrm{g}(\omega) \mathrm{d} \omega$

In amorphous $\mathrm{SiC}$, dynamical disorder corresponds to the following absorption spectrum $^{2}$

$\xi_{2}^{(\mathrm{d})}(\omega)=\mathrm{a}\left(\xi_{\mathrm{o}}-\xi_{\infty}\right) \gamma_{\mathrm{c}}\left\{\int_{\mathrm{R}}\left(\exp \left(-\mathrm{br}^{2}\right)\right)\right.$

$\left.\left[\frac{\omega_{\mathrm{TO}}^{3}}{\omega_{\mathrm{TO}(\mathrm{c})}^{2}\left(\omega_{\mathrm{TO}}^{2}-\omega^{2}\right)^{2}+\left(\gamma_{\mathrm{c}} \omega \omega_{\mathrm{TO}}^{1 / 2}\right)^{2}}+\frac{\omega_{\mathrm{LO}}^{3}}{\omega_{\mathrm{LO}(\mathrm{c})}^{2}\left(\omega_{\mathrm{LO}}^{2}-\omega^{2}\right)^{2}+\left(\gamma_{\mathrm{c}} \omega \omega_{\mathrm{LO}}^{1 / 2}\right)^{2}}\right] \mathrm{dr}\right\} \omega$

where $\omega_{\mathrm{LO}}$ is the angular frequency corresponding to the longitudinal optical mode; both $\omega_{\text {TO }}$ and $\omega_{\mathrm{LO}}$ depend on distance, $\mathrm{r}, \mathrm{R}$ being the r-domain.

Finally we shall consider very briefly amorphous Ge. It is well known that for this material we can assume $\xi_{2}(\omega)=\xi_{2}^{(s)}(\omega)$ (see ref. [2]). Then by combining eq. (2) with eq. (5) it is deduced: $\mathrm{g}(\omega)=\delta(\omega)$ (Dirac delta). In fig. 1 (see ref. [6]) we can observe the variation of $\omega \xi_{2}$ as a function of the wave-number for amorphous Ge. From our theoretical considerations, we have $\omega \xi_{2}(\omega)=K \omega \delta(\omega)$. This result agrees with fig. 1 in the range $0-100 \mathrm{~cm}^{-1}$.

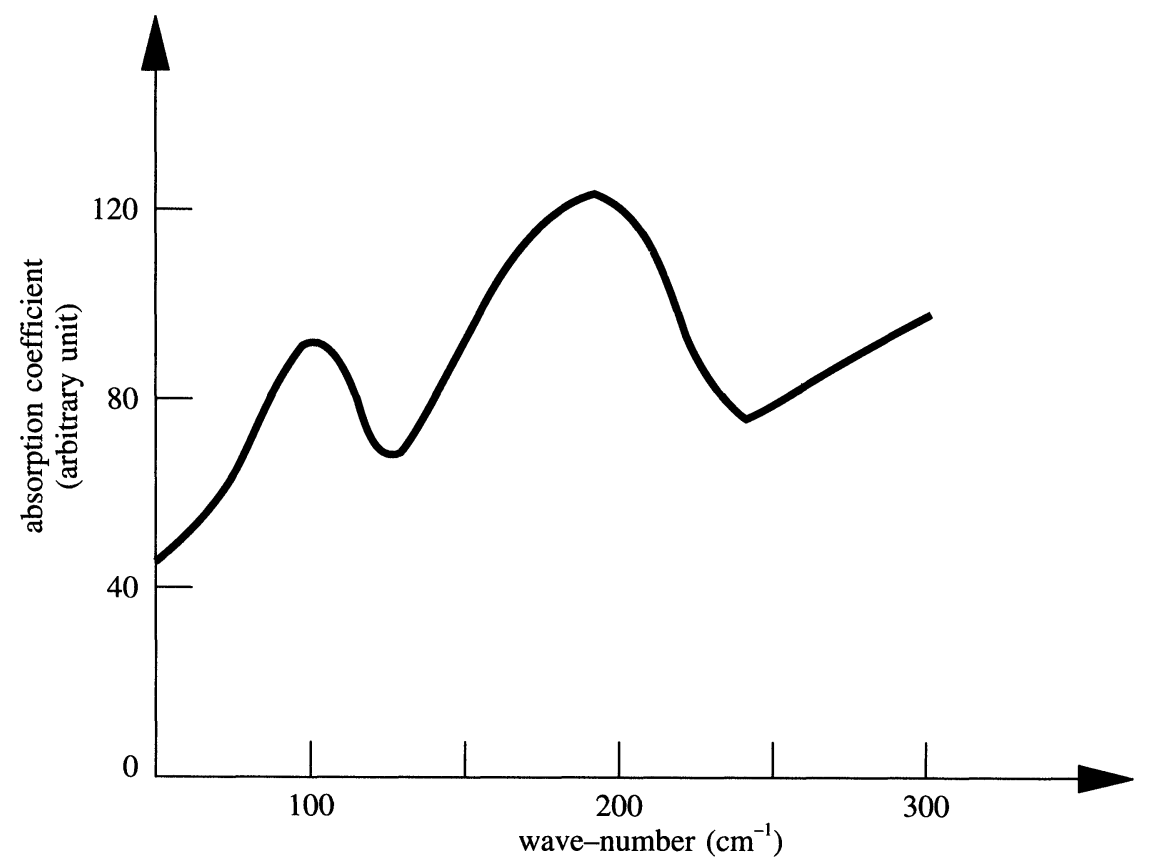

FIGURE 1 Experimental plot of the absorption coefficient as a function of the wave-number for amorphous Ge (after ref. [6]). 


\section{CONCLUSION}

Phonon density of states plays an important role in the context of amorphous solids. In particular, the relation between this density in amorphous III-V compounds and the no verification of the wave-vector conservation has been studied in various research works (see, for example, [6] [7]). This problem has been also considered in amorphous $\mathrm{SiC}$. This material has been investigated recently by several workers (see, for instance, ref. [8]) but the physical mechanisms associated with $\omega_{\mathrm{TO}}, \omega_{\mathrm{LO}}$ and dynamical disorder remain unclear. Finally, with respect to amorphous GaAs, eq. (7) is an important result.

\section{REFERENCES}

1. M.A. Grado and M. Grado. Phys. Lett. A 169, no. 5 (1992), 399-401.

2. S.S. Mitra, D.K. Paul and Y.F. Tsay. AIP Conf. Proc., no. 20 (1974), 284-289.

3. J.R. Hendrickson, U. Strom, P.C. Taylor and S.K. Bahl. AIP Conf. Proc., no. 20 (1974), 290296.

4. N.K. Hindley. J. Non-Cryst. Solids 5 (1970), 17.

5. H.K. Rockstad. AIP Conf. Proc. no. 20 (1974), 21.

6. R.W. Stimets, J. Waldman, J. Lin, T.S. Chang, R.J. Temkin and G.A.N. Connell. Solid State Commun. 13 (1973), 1485.

7. M.H. Brodsky and A. Lurio. Bull. Amer. Phys. Soc. 17 (1972), 322.

8. L. Magafas, N. Georgoulas, D. Girginoudi and A. Thanailakis. J. Non-Cryst. Solids 139 (1992), $146-150$ 

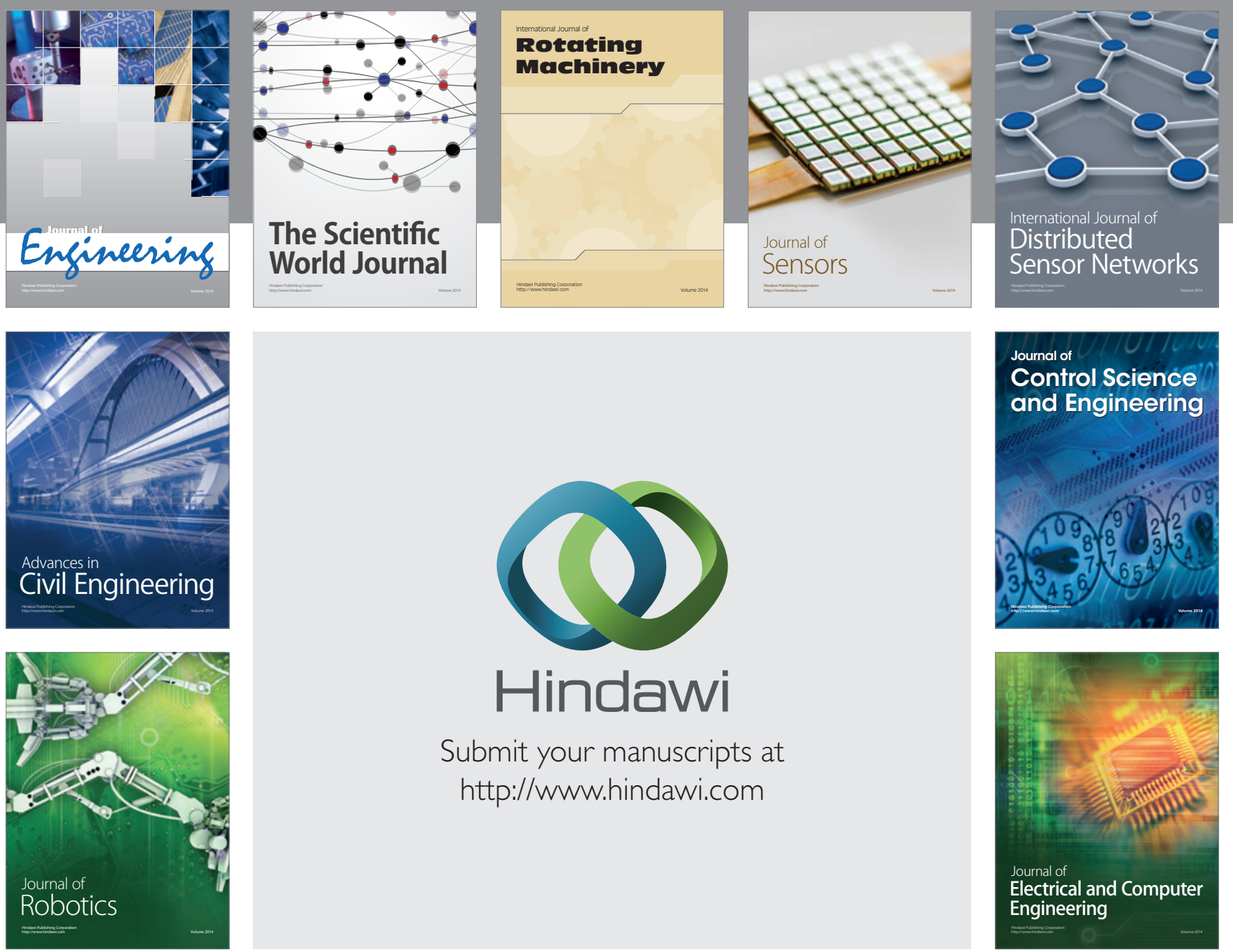

Submit your manuscripts at

http://www.hindawi.com
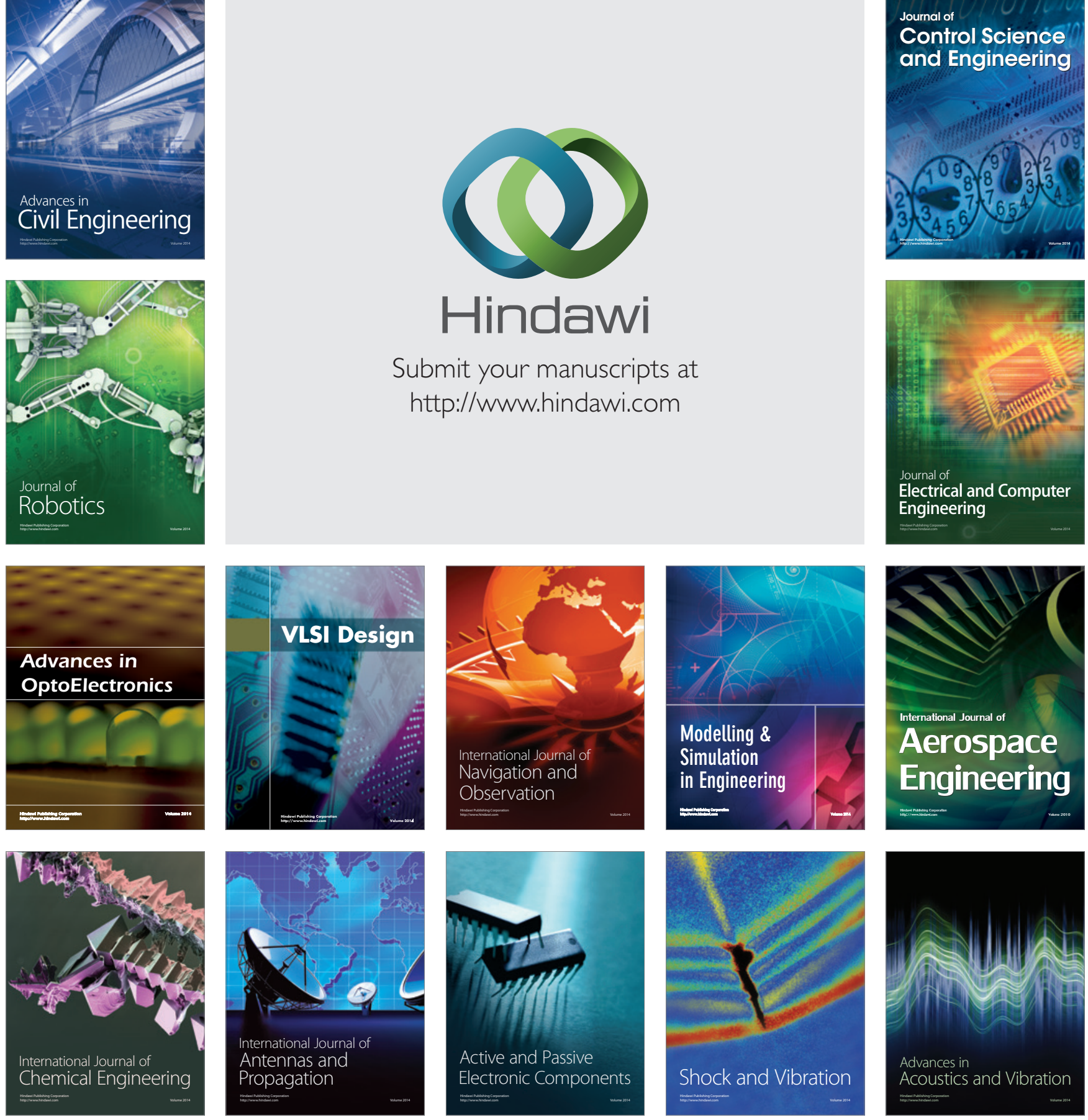